\title{
3D UNSTEADY FLOW SIMULATION IN AN OWC WAVE CONVERTER PLANT
}

\author{
A. EL MARJANI ${ }^{*}$, F. CASTRO ${ }^{* *}$, M. BAHAJI ${ }^{*}$, and B. FILALI ${ }^{*}$ \\ * Ecole Mohammadia d’Ingénieurs (EMI), University of Mohammed V Agdal, \\ Labo. de Turbomachines, Av Ibn Sina, B.P. 765 Agdal Rabat, Morocco, e-mail : marjani@emi.ac.ma \\ ** Depto. de Ingenieria Energética y Fluidomecánica. Escuela Técnica Superior de Ingenieros Industriales. \\ Universidad de Valladolid. Paseo del Cauce s/n, E-47011 Valladolid, España, e-mail : castro@eis.uva.es
}

\begin{abstract}
This paper deals with a numerical simulation to predict the air flow behaviour inside the chamber of an OWC wave converter plant by use of FLUENT code. The flow is assumed to be compressible, viscous and unsteady. Calculations have been conducted by considering a typical geometry (PICO plant) with an oscillating water free surface. In our analyses, we have only considered the case of monochromatic sinusoidal excitations with various frequencies and fixed amplitude. Analyses are focused on the flow behaviour characteristics both in the chamber and in the duct in order to obtain the temporal variation of velocity, pressure, flow rate and pneumatic power fields. Also, the mean characteristics like RMS ones of these parameters are determined. A great attention is paid to show the process of energy generation inside the chamber which can be exploited by a rotating turbine. In our case, we have included in the model a linear law relating the pressure drop to the mass flow rate in order to take into account the presence of the turbine. This is achieved in FLUENT by considering a domain of porous media in the turbine location. Simulation results obtained for all frequencies show that this code is very powerful and suitable for flow prediction in such geometries. Also, the amount of energy and also the aerodynamic losses between the chamber and the duct are well calculated.
\end{abstract}

\section{Key words}

Renewable Energy, Wave Energy, Oscillating Water Column, Unsteady Flow Simulation, Fluent Code.

\section{Introduction}

Energy in oceans encloses ocean thermal energy, tidal energy, wave energy and energy of marine currents. Great R\&D efforts have been made during the last decades, especially in the field of wave energy for conversion purpose to useful energy [1]. Developments are aimed to make exploitation of this kind of energy more attractive and more economical for possible commercial production.

Among the wide variety of possible wave energy systems converters, the Oscillating Water Column system (OWC) may be considered as the most used concept for ocean wave energy extraction, because it is a shoreline device and therefore cost installation is low and maintenance is easier as mentioned in reference ([2], [3]).

The process of energy conversion in such devices is based on the conversion of ocean incident wave's movement into an oscillating air flow inside an enclosed high volume chamber. A pneumatic energy is then generated following successive periodic air excitations due to the movement of the internal water free surface. The amount of energy which can be converted into useful energy depend strongly on the design of the geometry chamber. An important research is presented in reference [4] which is aimed to establish an optimisation strategy in different energy conversion stages. All parameters considered are global for example chamber dimensions. However, the model used in that reference doesn't incorporate any effect of aerodynamic losses which are encountered with air flow. These losses are directly dependent on the flow pattern inside the chamber and have an important effect on the global efficiency of the plant. No information about this point can be found in the researches reported in today's literature.

It is well known that the air flow is complex, particularly during phases of flow direction inversion. Flow is considered to be strongly unsteady and with variable regimes (laminar / turbulent) due to large velocity variation. Fluid compressibility play also important role on the plant performances as it has been reported in references ([4], [5]).

Importance of the chamber geometry is also underlined in the experimental study detailed in reference [6] in which analyses are aimed to find regimes of high movement amplification inside the chamber, especially those related to resonance occurrence. 
In the present study, an attempt aimed to improve the understanding of the local flow behaviour and also the global performances of an OWC device are performed with the help of the powerful FLUENT code. Objectives are connected to the elaboration of a model for air flow simulation in the chamber. The obtained results will be exploited in the design stage of a new project of an OWC wave energy converter.

We mention here that these researches are conducted as part of the common project between fluid mechanic and turbomachinery research teams respectively of the University of Valladolid (Spain) and the University of Mohammed V-Agdal (Morocco) for the development of a new project of an OWC converter plant.

\section{OWC system operation}

An OWC device consists mainly of a submerged chamber of air connected to atmosphere by means of a circular duct inside which a bidirectional flow turbine is installed (see figure 1). The flow rate of air through the turbine is generated by successive incident sea water waves that compress and depressurize air in the chamber by the periodic motion of the oscillating free surface.

Several kinds of turbines have been envisaged and used for pneumatic energy conversion to mechanical energy. The most used ones correspond to the Wells turbine [2] which is of axial type and the impulse turbine which is of symmetrical blades, originally used in steam power plant applications ([5], [8]). Investigations reported in today's literature reveals the existence of adaptability problems and more R\&D efforts are needed to adapt a suitable and efficient turbine.

Several full size pilot plants based on the OWC principle have been built in the world as mentioned in references ([2], [7]). We can mention for example:

- $\quad$ PICO plant in Azores for a power of $400 \mathrm{~kW}$.

- LIMPET plant in Islay Island for a power of $500 \mathrm{~kW}$.

- Port Kembla, NSW, plant in Australia for a power of 200/300 kW.

- TRIVANDRUM plant in India for a power of $150 \mathrm{~kW}$.

- $\quad$ SAKATA plant in Japan for a power of $60 \mathrm{~kW}$.

- OSPREY plant in Scotland for a power of 2 MW ( destroyed at present ).

In order to prevent excessive flow rate through the turbine, a control valve can be installed in the top of the chamber ( Figure 2 ) as is the case of PICO plant. It is shown that the Wells turbine performances are improved by the presence of this element [2].

In the present study, we don't take into account the influence of the valve. Simulations are performed without this element. It is planed to be introduced later.

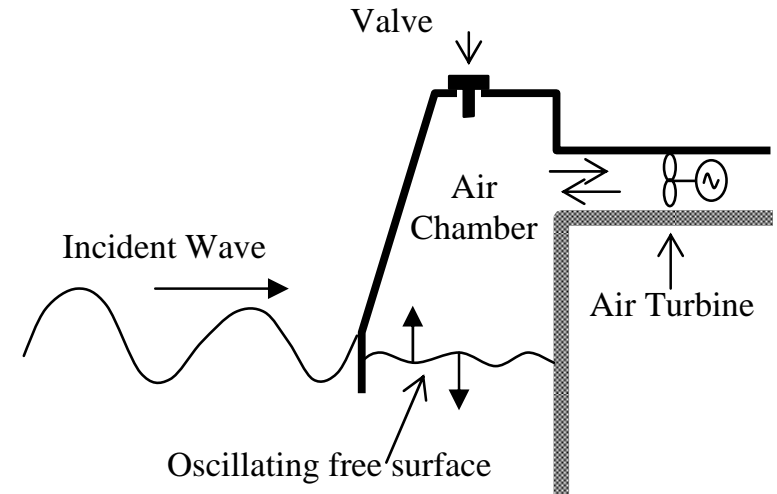

Fig. 1. The oscillating water column system

\section{Flow simulation in the air chamber}

As mentioned before, the aim of this study is to analyse the flow behaviour inside the chamber of an OWC plant. Analyses are conducted by use of FLUENT code. The main geometric characteristics of the chamber are depicted in figure 2. Dimensions correspond to those of the PICO plant built in Azores [3]. We have chosen this plant in order to test the applicability of FLUENT code to predict flow in similar configuration.

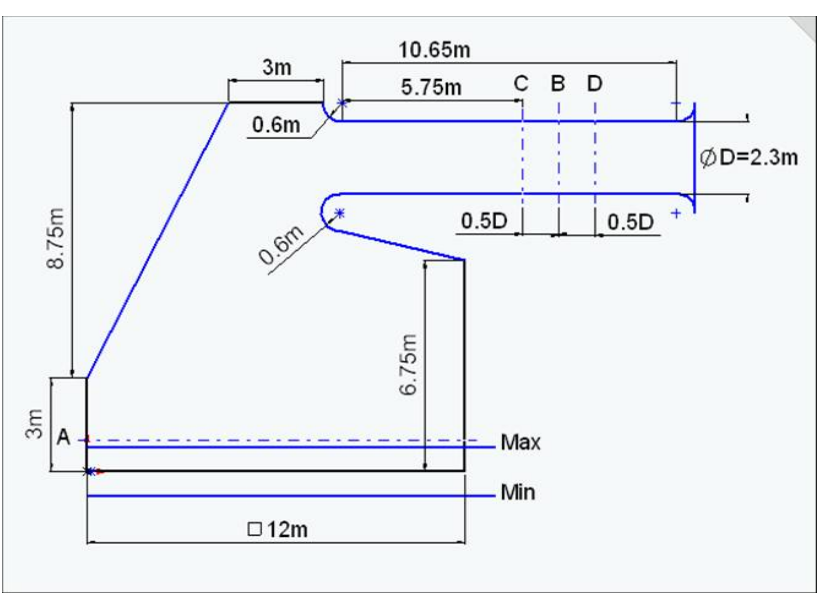

Fig. 2. Main dimensions of the geometry

In this configuration flow is considered viscous, 3D and unsteady with compressibility effects. The code is used with a solver of coupled conservation equations (continuity, momentum and energy), with an implicit time scheme and with a dynamic mesh in the area of moving boundary (oscillating free surface in figure 1). Turbulence is modelled with the classical two equations $(\mathrm{k}-\varepsilon)$ model. The system of conservation equations is closed by the classical state equation for an ideal gas.

Boundary conditions adopted correspond to a fixed pressure at the outlet of the duct (atmospheric pressure) and a sinusoidal moving boundary at the oscillating free surface. In the rest of the geometry, conditions of zero velocities on walls are applied. 
Due to the oscillating movement of the water free surface, we have fixed in numerical calculations a time variation velocity in this location by using the following equation:

$$
v(t)=H \cdot\left(\frac{2 \pi}{T}\right) \cdot \cos \left(\frac{2 \pi}{T} t\right)
$$

( $v$ : velocity, $T$ : period, $t$ : time, $H$ : height amplitude )

The water surface displacements associated with the integration of equation (1) vary between levels indicated by "Min" and "Max" in figure 2.

The model is completed by the influence of a turbine which is supposed located at section B (figure 2). We have considered a turbine of Wells type which is characterized by a linear relation between the stagnation pressure change and the mass flow rate ([2], [4]). In our case, this characteristic is depicted in figure 3.

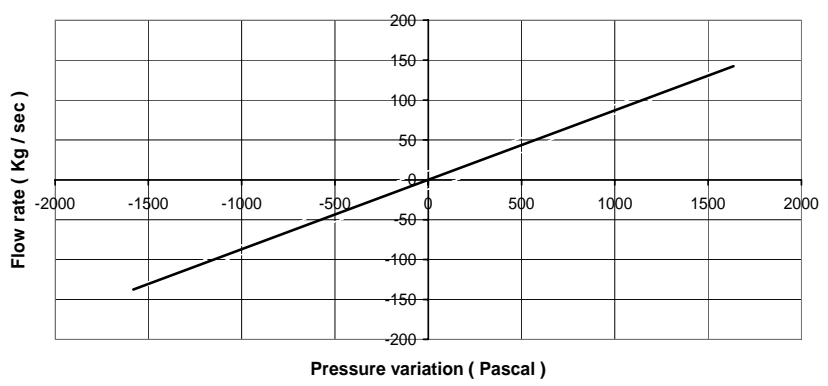

Fig. 3. Turbine characteristic

Note that the pressure change through the turbine is calculated as the difference between pressures at sections $\mathrm{C}$ and $\mathrm{D}$ (figure 2). We have chosen locations at $1 / 2 \mathrm{duct}$ diameter on both sides from the turbine location.

The introduction of the turbine characteristic in FLUENT is achieved by setting a porous medium in this area. For such medium, the pressure change is governed by the Darcy's law as following:

$\Delta p=-\left(\frac{\mu}{\alpha} v+\frac{1}{2} C \rho v^{2}\right) \cdot \Delta m$

( $\Delta p$ : pressure change, $\mu$ : laminar viscosity, $\alpha$ : medium permeability, $v$ : velocity normal to the porous face, $\Delta m$ : medium thickness, $C$ : pressure-jump coefficient).

In order to ensure linear variation we have cancelled the constant $C$ and adjusted the others parameters in equation (2) in order to fit the desired turbine characteristic.

GAMBIT associated with FLUENT code is used for meshing the 3D geometry. Mesh in the area of moving surface ("Min" to "Max" in figure 2) consists of quadrilateral cells, in the rest of the domain triangular ones are used (see figure 4). The mesh used in all calculations is characterized by a total of 1275 nodes, 3897 cells and 8749 faces.

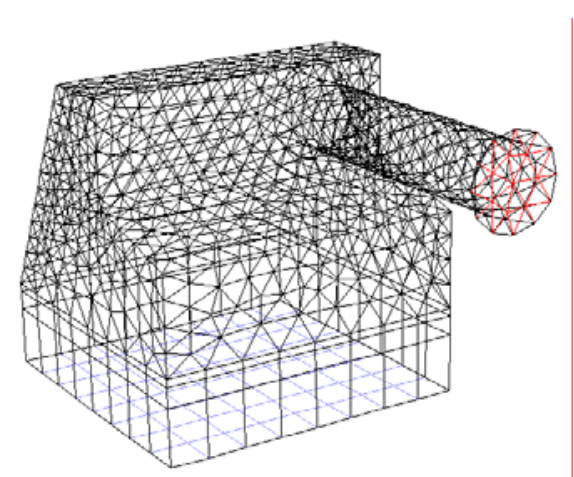

Fig. 4. 3D geometry with mesh on boundaries

\section{Results}

In a first stage, calculations are performed by considering a monochromatic sinusoidal variation (equation 1) for the water moving boundary with various periods: 6-8-1012-16 sec. Unsteady calculations are performed for 4 successive cycles and more in some cases in order to eliminate transient effects which are encountered in the first time steps. We have adopted for all considered periods a time step of $0.01 \mathrm{sec}$. The amplitude of the height $\mathrm{H}$ (equation 1 ) is equal to $0.78 \mathrm{~m}$ [9].

In the following, we present first results corresponding to the instantaneous predicted characteristics such as: velocity, stagnation pressure, pneumatic power for the period of $6 \mathrm{sec}$ in sections A, C and D. Second, we present results of calculated RMS values for these characteristics for all periods. Also the evolution of losses with frequency variation will be discussed. The sections A, C and D refers to those mentioned in figure 2 . Note that section A is located very close to the maximum level of height indicated by "Max".

\section{A. Instantaneous characteristics $(T=6 \mathrm{sec})$ :}

In figure 5 is depicted the time velocity variation for station $\mathrm{C}$ and for the oscillating water surface which has been amplified by 10 due to its low level. It can be seen that the velocity reaches a maximum value of $30 \mathrm{~m} / \mathrm{sec}$. Also comparison of curves reveals the existence of a dephasing of about $0.38 \mathrm{sec}$ which may be associated to the compressibility of air.

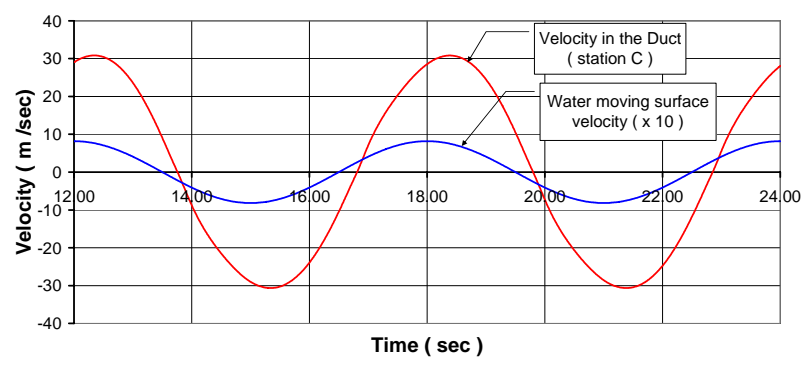

Fig. 5. Time velocity variation

In figure 6 we present the time variation of the stagnation pressure with respect to atmospheric pressure at stations A, C and D. Pressure variation at station D are very low due to its location which is close to the duct outlet or entrance ( depending on flow direction ). The maximum 
pressure generated inside the chamber is about 0.3 bar which is actually not high. So effect of compressibility may be neglected in this domain.

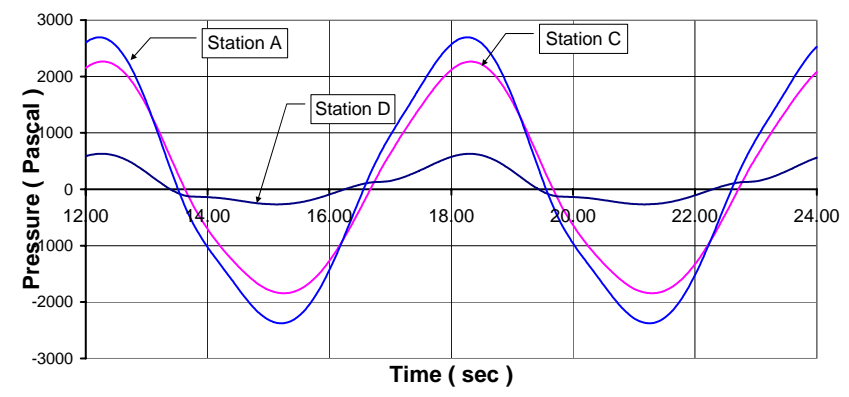

Fig. 6. Time stagnation pressure variation

Figures $7 \mathrm{a}$ and $\mathrm{b}$ indicate the temporal pneumatic power variation respectively stored inside the chamber and the duct. This quantity is calculated as following:

$$
P=\frac{1}{\rho} \Delta p_{0} \cdot Q_{m}
$$

Where $\Delta p_{0}$ is the stagnation pressure difference, $\rho$ is the air density and $Q_{m}$ is the mass flow rate. For the chamber $\Delta \mathrm{p}_{0}$ is calculated between any station (A or C or D) and outside of the air chamber. Predicted results indicate that the pneumatic power can reach a significant maximum value of $300 \mathrm{~kW}$ in the chamber. Evidently the power inside the duct is lesser than in chamber due to losses in stagnation pressure. In station D the power level is the lowest due to the drop of pressure through the porous medium.

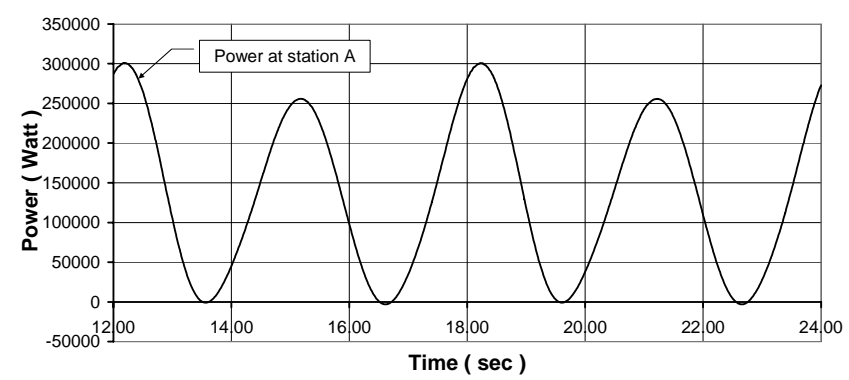

Fig. 7 a. Pneumatic power inside the chamber

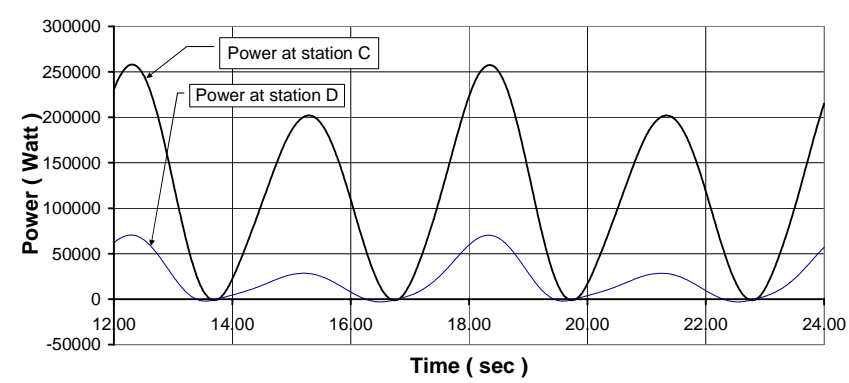

Fig. 7 b. Pneumatic power inside the duct

We mention that the analysis of the predicted results obtained for the others periods have similar time variation, only differences are noted in reached level for all characteristics.

\section{B. RMS characteristics :}

In order to facilitate analyses of temporal variation, we have calculated time means values based on the root mean square (RMS) calculation. For any flow characteristic designed by $\mathrm{x}(\mathrm{t})$ varying with time, the corresponding RMS value $\mathrm{X}$ over a period $\mathrm{T}$ is calculated by the following relation:

$$
X=\sqrt{\frac{1}{T} \int_{0}^{T} x^{2} d t}
$$

First of all we have been interested in the evolution of the RMS values of velocity and mass flow rate inside the duct by varying frequency. It has been observed that these two quantities have linear variation with frequency.

In order to determine the evolution of losses between the chamber (station A) and the duct (station C) RMS values of stagnation pressure and power are needed. Figures 8 and 9 indicate the evolution of these quantities. It can be observed that the differences for both pressures and powers grow with frequency. This is better illustrated in figures 10 and 11 in which we have plotted the drop of pressure and power versus frequency. Also these figures reveal that pressure drop variation is well fitted by a parabolic relation and power drop variation by a cubic relation. This losses behaviour is normal owing to the fact that aerodynamic pressure losses in general are proportional to the square of flow rate.

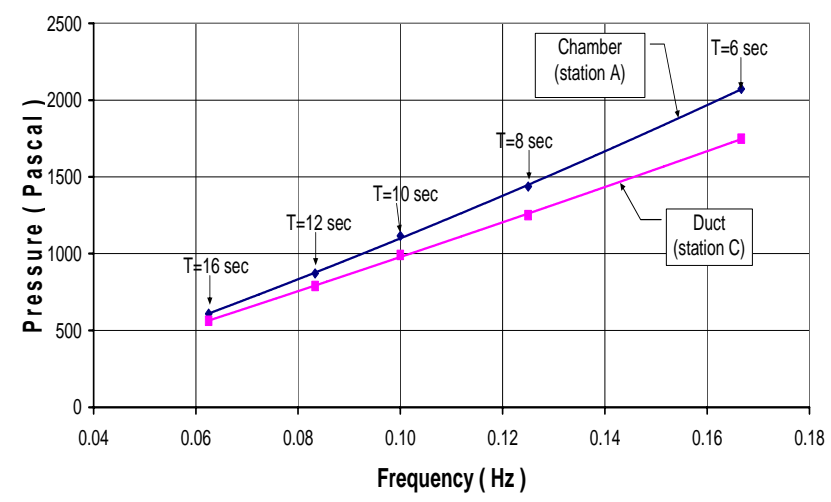

Fig. 8. Stagnation pressure vs. frequency

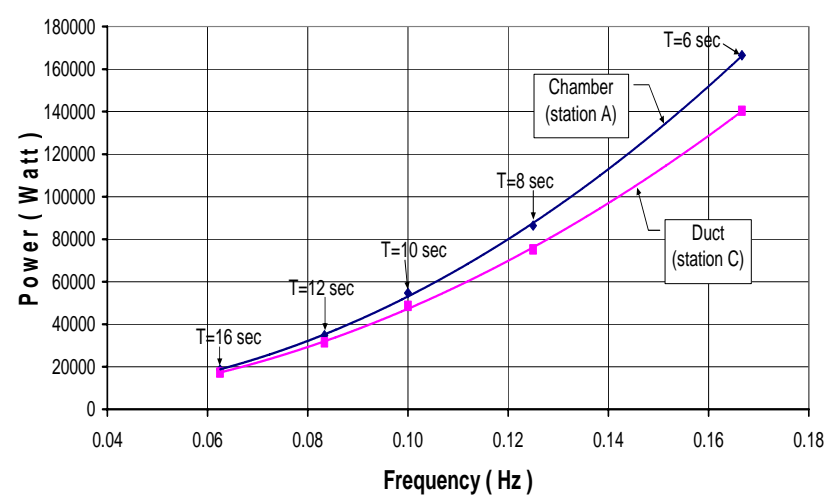

Fig. 9. Pneumatic power vs. frequency 


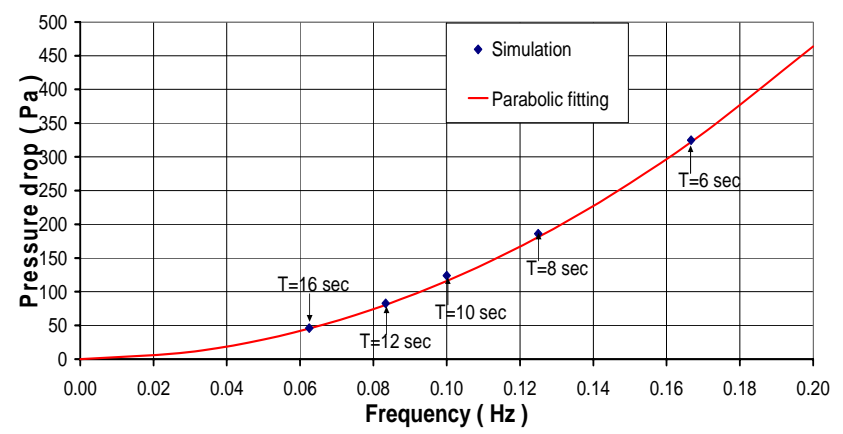

Fig. 10. Stagnation pressure drop between stations A and C

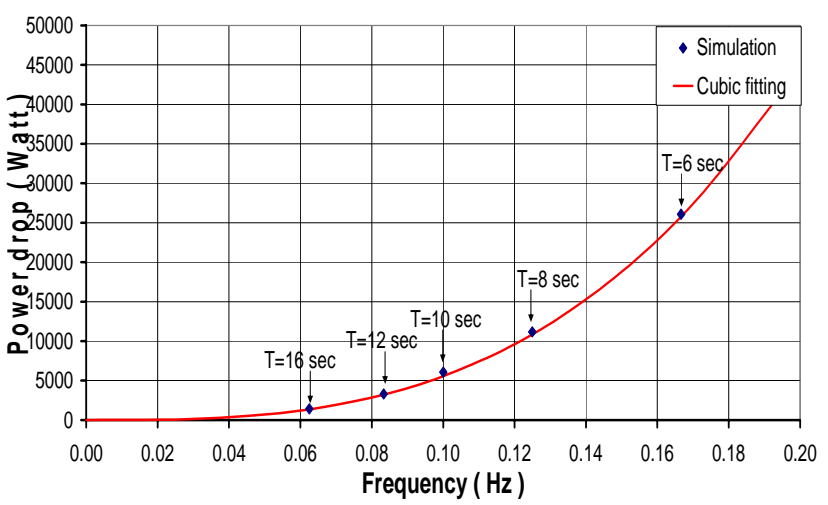

Fig. 11. Power drop between stations A and C

Finally the mean pressure losses coefficient $\xi$ is plotted in figure 12. This parameter is defined by:

$\xi=\frac{\left(p_{0}\right)_{A}-\left(p_{0}\right)_{C}}{\rho \cdot v_{C}^{2}}$

$\left(p_{0}\right)_{A}$ or $\left(p_{0}\right)_{C}$ : RMS stagnation pressure at section $\mathrm{A}$ or $\mathrm{C}$ and $v_{c}{ }^{2}$ : RMS velocity at section $\mathrm{C}$.

This coefficient is practically constant and its mean value lies between 0.5 and 0.6 , which is a good calculation since $\xi$ is a characteristic of the geometry and its value depends only on its form.

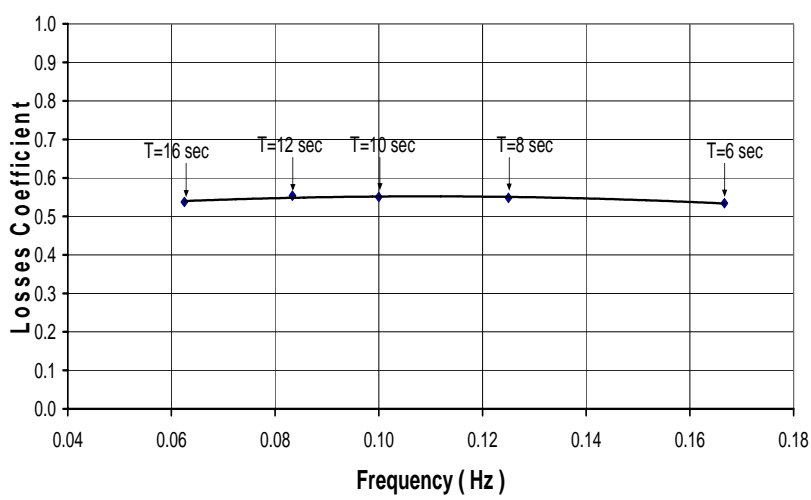

Fig. 12. Losses coefficient between stations A and C

\section{Temporal fields of pressure and velocity :}

Figures 13 a, b, c and d indicate the temporal pressure variation inside the domain of calculation. Results reveal that large pressure variations are registered in the duct especially near the turbine location. Compression and depressurization phases are mentioned in all figures.
Pressure waves propagation process through the duct can be clearly observed. Waves are of plane type.
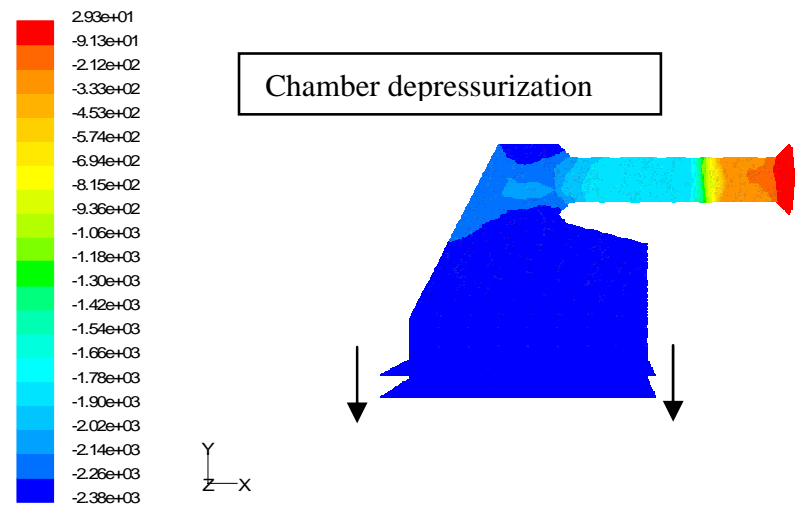

Fig. 13 a. Pressure variation ( $1 / 4$ of period )

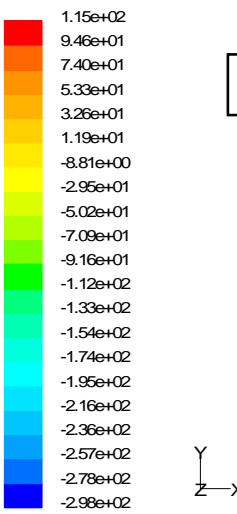

\section{Chamber depressurization}

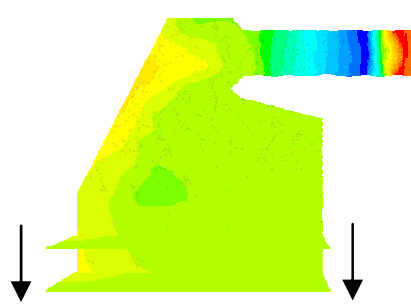

Fig. 13 b. Pressure variation ( $1 / 2$ of period )

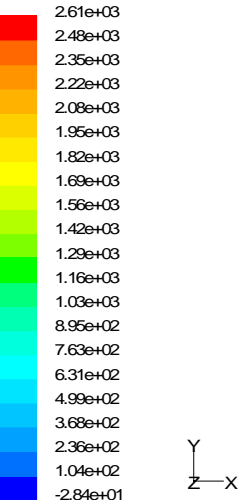

Chamber compression

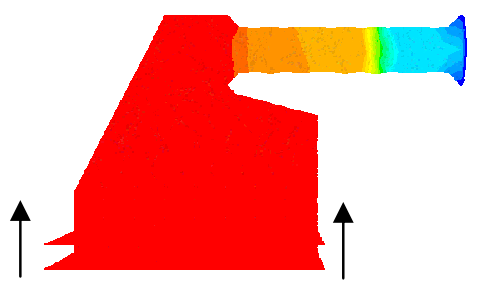

Fig. 13 c. Pressure variation ( $3 / 4$ of period )

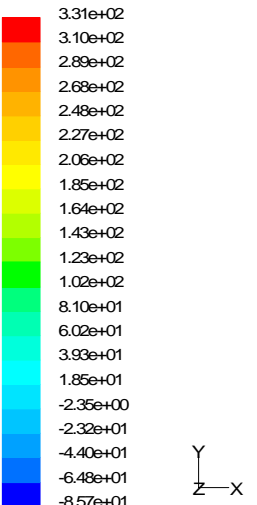

Chamber compression

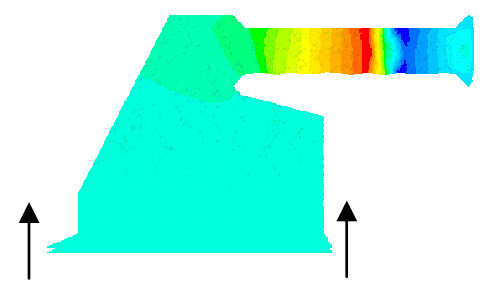

Fig. 13 d. Pressure variation ( one period ) 
Figures 14 a, b, c and d indicate the temporal velocity variation inside the domain of calculation. Results reveal that the whole domain is concerned by large variations. We note that the largest velocity variation in the chamber occur during the depressurization phase. High gradient can be observed which affect strongly losses.

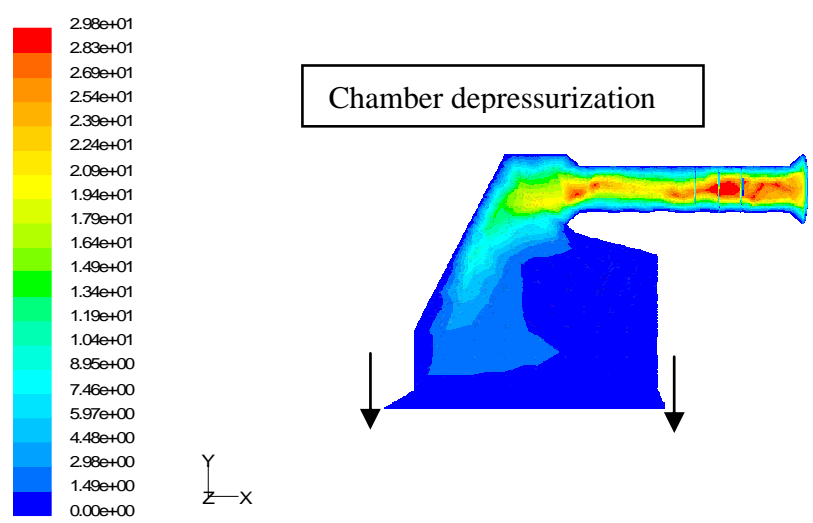

Fig. 14 a. Velocity variation ( $1 / 4$ period )
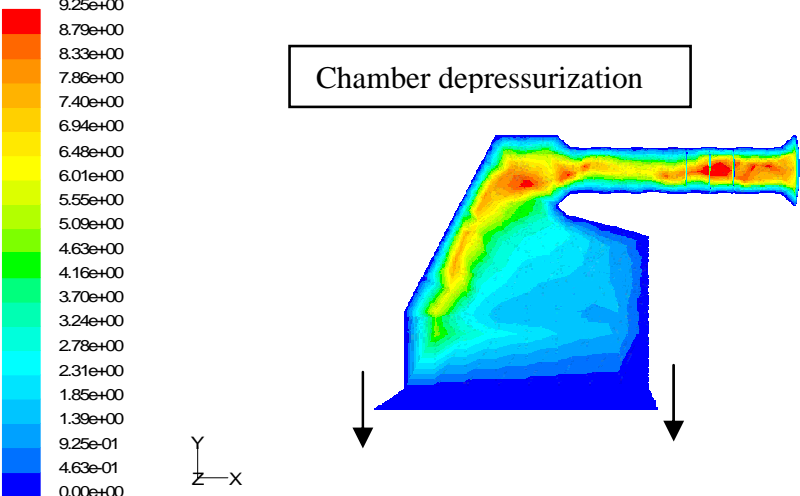

Fig. 14 b. Velocity variation ( $1 / 2$ period )

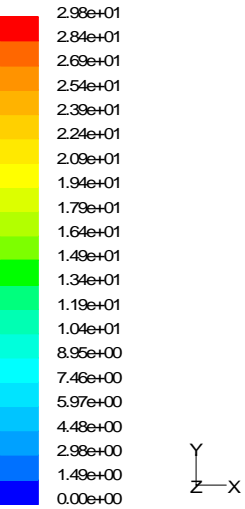

$$
\text { Chamber compression }
$$

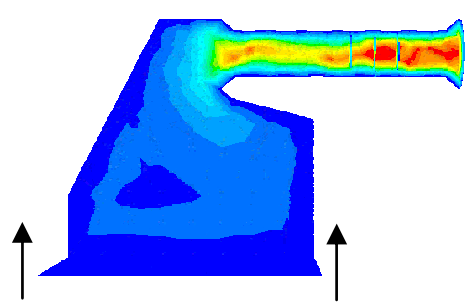

Fig. 14 c. Velocity variation ( $3 / 4$ period )

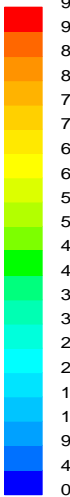
$9.97 \mathrm{e}+00$ $9.47 \mathrm{e}+\infty 0$ $8.97++\infty$ $8.47 \mathrm{e}+00$ $7.98++\infty 0$ $7.48 \mathrm{e}+\infty 0$ $6.98 \mathrm{e}+\infty 0$ $6.48 \mathrm{e}+\infty 0$ $5.98++\infty 0$ $5.48 \mathrm{e}+00$ $4.99++\infty$ $4.49++00$ $3.99 \mathrm{e}+00$ $3.49 \mathrm{e}+00$ $2.99++\infty 0$ $249 e+\infty 0$ $2.49 e+00$ $1500+\infty$ .97e-0 $4.990-01$
$0.000+00$

Fig. 14 d. Velocity variation ( one period )

\section{Conclusions}

3D Simulations of unsteady flow in an OWC chamber are performed with FLUENT code. Results indicate that this code is powerful and well suitable to predict the flow characteristics in such configuration. Simulations are conducted for different periods which generate various mass flow rates and amounts of power. Calculations reveal that high level of pneumatic energy can be reached. In this study we have tested with success the model of porous media to simulate the turbine characteristic. Also the behaviour of losses is well predicted. With this tool the unsteady flow behaviour can be analysed step by step in order to detect characteristics distortions and regions of high losses. This can be exploited in the stage of the design of a new plant.

Calculations using a probabilistic mathematical law for the moving free surface are now envisaged in this study.

\section{Acknowledgments}

Authors want to thank the Spanish AECI, Cátedra de Energías Renovables at the University of Valladolid, the Direction of EMI and the Presidency of University of Mohammed V-Agdal for supporting this research.

\section{References}

[1]. A. CLEMENT et al. : "Wave Energy in Europe : Current status and perspectives" Renewable \& Sustainable Energy Reviews 6 (2002) 405-431

[2]. A. F. de O. FALCÃO : "R\&D Requirements for Fixed Devices" WaveNet, Results from the work of the European Thematic Network on Wave Energy, March 2003.

[3]. A. F. de O. FALCÃO : "The shoreline OWC wave power plant at the Azores" Proced. Of the Fourth European Wave Energy Conference, Aalborg, Denmark, December 2000 ( paper B1 ).

[4]. J. W. WEBER, G. P. THOMAS : "An investigation into the Importance of the Air Chamber Design of an Oscillating Water Column Wave Energy Device" ISOPE 2001, Staranger, Norway.

[5]. A. TAKKER, T. S. DHANASEKARAN : "Effects of Compressibility on the Performance of a Wave Energy Conversion Device with an Impulse Turbine Using a Numerical Simulation Technique" Int. J. of Rotating Machinery, 9 : 443-450, 2003.

[6]. R. H. TSENG, R.-H. WU, C.-C. HUANG : "Model study of a shoreline wave-power system" Ocean Engineering 27 (2000) 801-821.

[7]. Marine Energy Challenge, Oscillating Water Column Wave Energy Converter Evaluation Report, The Carbon Thrust, 2005.

[8]. T. S. DHANASEKARAN, A. TAKKER : "Threedimensional effects in computational fluid dynamic analysis of impulse turbine for wave energy conversion", ISSEC 2003 Symposium

[9]. C. JOSSET : "Simulation numérique d'une centrale houlomotrice par une méthode couplée Rankine/Kelvin". Thèse Centrale Nantes/Université de Nantes 2002. 